\title{
Los diarios de Roland Barthes
}

\author{
Roland Barthe's Diaries
}

\author{
Ángeles Sirvent Ramos \\ Universidad de Alicante \\ ma.sirvent@ua.es
}

\begin{abstract}
The unpublished texts of a writer, of an intellectual, are always very revealing, and their publications pleasantly welcomed. In this sense, having unveiled, in this 21 st century, unpublished texts of Roland Barthes, such as journal entries, are of great value, not only because Barthes had decreed the need for the "death of the author" and not just to know details and personal reflections, but because through them the intimate writing of Roland Barthes is revealed, as well as his hesitations and his projects of future writing.

In this article, we show the intrinsic value that these texts -written on notebooks or files- possess, but also, and after observing the drafts of their future project Vita Nova, we let them intuit the use of them in such work; project truncated almost from the beginning because of his premature death.
\end{abstract}

\section{Key-words}

Roland Barthes, Diary, Incidents, Carnets $d u$ voyage en Chine, Journal de deuil.

\begin{abstract}
Résumé
Les textes inédits d'un écrivain, d'un intellectuel, relèvent toujours d'un grand intérêt, et leur publication est toujours très bien accueillie. Avoir publié, dans ce XXIe siècle, des textes inédits de Roland Barthes, tels les notations d'un journal personnel, a plus de valeur, non seulement parce que Barthes avait décrété le besoin de la "mort de l'auteur", non seulement parce que nous apprenons des détails et des réflexions personnelles, mais parce que ces fragments révèlent l'écriture intime de Barthes, ainsi que ses hésitations et ses projets pour une nouvelle écriture.

Nous faisons remarquer la valeur en soi de ces textes, écrits en des carnets ou sur des fiches, mais aussi, et après avoir révisé les brouillons de son futur projet Vita Nova, il nous est permis d'affirmer que ces fragments auraient fourni le matériel de certains chapitres de cette œuvre, projet tronqué presque dès son début à cause de la mort prématurée de Barthes.
\end{abstract}

\section{Mots-clés}

Roland Barthes, Journal intime, Incidents, Carnets du voyage en Chine, Journal de deuil. 
Puede sorprender a aquellos que no han seguido la trayectoria última del pensamiento y la escritura barthesiana hablar aquí de una escritura tan íntimamente ligada al yo como es el diario. Barthes es mucho más que el estructuralista, que el semiólogo, incluso que el postestructuralista, que el propulsor de la "muerte del autor". El propio Barthes mostró tres años después de su famosa y trascendente proposición cómo el autor podía alojarse en el texto, en la escritura; mediante los biografemas el autor quedaba invitado a formar parte de uno de los códigos más del texto. Y ese proyecto autobiografemático Barthes lo llevó él mismo a escena en su conocido y delicioso Roland Barthes par Roland Barthes.

Barthes llevó a lo largo de su vida una especie de diario. Acostumbraba a anotar, como Gide ${ }^{1}$, sus reflexiones, sus proyectos, sus impresiones sobre las lecturas, en un carnet, principalmente en fichas de un formato de un cuarto de A4. La enorme cantidad de fichas -que ignorábamos; hay millares de ellas-, supusieron, como veremos, una fuente para las obras que vendrían, el material para la obra futura.

Tiphaine Samoyault, una de las pocas investigadoras a las que se ha permitido el acceso al amplio "Grand Fichier" - como a las "Agendas"- de los documentos inéditos de Barthes, lo define como "l'instrument de travail privilégié de Barthes, qui prend parfois l'allure d'un journal” (2015b: 43). De todo este material inédito se obtendrán las páginas que se publicarán años después de la muerte de Barthes y que nos permitirán conocer el diario que, aunque fragmentario, en la forma y en el tiempo, había acompañado a nuestro autor a lo largo de su existencia.

En vida de Barthes pudimos tener acceso a pequeños fragmentos de un diario discontinuo. Nos referimos a "Délibération". Barthes lo publica en la revista Tel Quel el invierno de 1979. Tras reflexionar sobre el hecho de llevar a cabo un diario y sobre todo -de ahí el título del artículo- de la pertinencia de su publicación, se plantea además la necesidad de transgredir este género literario. Como expresa Éric Marty, se trataría de ir del "journal-genre" al "journal-texte": "Barthes se place tout à fait -écrit Marty²- dans une démarche antigénérique ${ }^{3}$ qui est celle de la position moderne: moderne, au sens où la modernité a suscité une tradition antigénérique [...] qu'il [le journal] soit capté par la catégorie transcendantale de l'écriture" (2007: 87).

1 Para un estudio de las similitudes intelectuales y personales entre estos dos autores, ver la obra de J. Benito Fernández (2011).

2 A quien Barthes dedica este artículo, encontrándose Marty en ese momento, tal como él mismo nos confiesa, llevando a cabo su tesis sobre el Journal de Gide (Marty, 2007: 89). Marty publicará en 1985, en las ediciones du Seuil, L'écriture du jour: le 'Journal'd'André Gide, que recibirá el Gran Prix de la critique. Recordemos igualmente que el primer artículo que publicó Barthes - dejando de lado "Culture et tragédie", publicado en los Cahiers de l'étudiant, en la primavera de ese mismo año- trata precisamente del Diario de Gide: "Notes sur André Gide et son journal" (1942). Y que en una de las últimas sesiones de su curso en el Collège de France antes de morir, la del 19 de enero de 1980, sigue recordando a Gide y su diario.

3 Gérard Genette avait déjà considéré cet article un 'antijournal' (1981). 
De hecho, Barthes está pensando en el diario como práctica ya en la época del Roland Barthes par Roland Barthes (1975). Tras la aparición de retazos de su escritura diarística, cada una de las líneas de uno de los fragmentos de dicha obra cobra una importancia particular. En su "Du fragment au journal”, Barthes escribe:

Sous l'alibi de la dissertation détruite, on en vient à la pratique régulière du fragment; puis du fragment, on glisse au "journal”. Dès lors le but de tout ceci n'est il pas de se donner le droit d'écrire un "journal"? Ne suis-je pas fondé à considérer tout ce que j'ai écrit comme un effort clandestin et opiniâtre pour faire réapparaître un jour, librement, le thème du "journal" gidien (1975: 99).

Y es así ¿No es ya Roland Barthes par Roland Barthes una preciosa muestra de su espacio autobiográfico personal e intelectual ${ }^{4}$ ? ¿No se acerca el diario, esta notación del presente, esta escritura fragmentaria, a los "biografemas" ya enunciados por Barthes en su Sade, Fourier, Loyola? (1971) ¿No veremos trazos en esta escritura diarística del "punctum” de La chambre claire? (1980).

Del mismo modo encontramos en este fragmento del Roland Barthes par Roland Barthes sus precauciones ante tal género, ya en descrédito -como enuncia tanto en este fragmento como en el artículo "Délibération"-, en el que, además, pone el término "diaire" en correlación con "diarrhée et glaire” (1975: 99), en la línea en que Valery Larbaud denominó sus "Borborygmes". El autor de "la muerte del autor" teme la "contemplation de mes déchets (narcissisme)" (1975: 99), como en "Délibération" muestra su precaución ante "l’hémorragie de l'Imaginaire" (2002a: 680).

Aun desde dicho marco reflexivo, Barthes nos regala dos muestras de su escritura diarística de los últimos años. La primera corresponde a fragmentos escritos en "U.5" dos años antes, del 13 de julio al 13 août 1977, durante la enfermedad de su madre. Este 'Journal d'Urt', como nos gusta denominarlo, muestra la vida cotidiana de Barthes en le Suroeste francés: las costumbres, las compras, la comida: "La course du matin (chez l'épicier, le boulanger, alors que le village est encore presque désert), je ne la manquerais pour rien au monde" (2002a: 672).

El tiempo, los placeres, las sensaciones están presentes: “Ce matin, au sein du Souci, un isolat de bonheur: le temps (très beau, très léger), la musique (du Haydn -aunque preferirá siempre Schumann-), le café, le cigare, une bonne plume, les bruits ménagers..." (2002a: 674), las lecturas de esos momentos: los Cahiers de la Petite Dame, Chateaubriand, Suzuki, Blanchot hablando de Proust, Guerre et paix. Pero también las "sombres pensées": "Sombres

4 A estos efectos se ha encaminado mi entrada "Roland Barthes par Roland Barthes", en el Diccionario Barthes que prepara Claude Coste para las ediciones Honoré Champion.

5 Por Urt, su casa de vacaciones, y al mismo tiempo de trabajo, cerca de Bayona. Con motivo del centenario del nacimiento, esta ciudad rindió a Barthes un amplio homenaje del 15 al 17 de septiembre de 2015.

http://www.bayonne.fr/fileadmin/user_upload/fichiers/Mairie_de_Bayonne/Espace_presse/Dossier\%20de $\% 20$ presse_Roland $\% 20$ Barthes $\% 20 \% \mathrm{C} 3 \%$ A $0 \% 20$ Bayonne.pdf 
pensées, peurs, angoisses: je vois la mort de l'être cher, m'en affole, etc.” (2002a: 671), y la famosa frase escrita el 13 de agosto de 1977: "Tout d'un coup, il m'est devenu indifférent de ne pas être moderne" (2002a: 676).

Estos fragmentos están seguidos de la inscripción, dos años después, el 25 de abril de 1979, de la "Vaine soirée" de la víspera en París. Su deambular por las calles de París bajo una fría lluvia, el vacío que siente ante la ciudad y las personas, incluso en la inauguración de la exposición de "D.B.", del que aprecia no obstante las fotografías ${ }^{6}$, su paso por el Flore: "aucun visage auquel s'intéresser ou sur quoi fantasmer, ou tout au moins fabuler", que hace seguir de unas importantes líneas que aluden a su proyecto en ciernes, desgraciadamente truncado: “L'échec lamentable de la soirée m'a poussé à essayer d'appliquer enfin la réforme de vie que j'ai en tête depuis longtemps. Ce dont cette première note est la trace" (2002a: 677).

Tras cuestionarse pues la oportunidad de la publicación de un diario, está llevando a cabo al mismo tiempo la publicación efectiva de fragmentos de su escritura diarística, y lo hará, además, en una revista de vanguardia, como es Tel quel.

Diríamos que a través de esos dos tipos de textos, no sólo de temática bien diferente, sino con una diferente organización textual, Barthes lanza una prueba para testar la acogida de los textos del artículo. El diario de Urt que reproduce está presentado bajo la forma de pequeños fragmentos; el texto de la Vaine Soirée es un texto ya más desarrollado. Una nueva ‘deliberación' se presenta a nuestro entender ante Barthes, en cuanto a la forma de ese posible diario: ¿continuo? ¿fragmentario? Es en el fondo la misma vacilación que muestra Barthes ante la forma con que debería acometer su nueva producción.

¿Fragmentos de este tipo, anotaciones de un diario íntimo, son publicables? Tras estos dos textos, Barthes se plantea la escritura del diario, de un diario sin "egotismo", y la "publicabilidad" ocupa de nuevo las reflexiones de Barthes en las últimas páginas de "Délibération", dejando sólo la puerta entreabierta en su decisión.

François Wahl, editor en aquel momento de Barthes en las ediciones du Seuil, lo llevará a cabo tras la muerte de Barthes, y con textos bien comprometedores. Se trata, como sabemos, de la serie Incidents (1987).

Wahl justificará dicha publicación póstuma (1987: 8-9) por el proyecto anunciado y enunciado en el "Projet de livres" del Roland Barthes par Roland Barthes: "Incidents (mini-textes, plis, haïkus, notations, jeux de sens, tout ce qui tombe, comme une feuille" (Barthes, 1975: 153).

¿A Barthes le hubiera gustado esta publicación, o, al menos en la forma y el momento en que se presentan? Siempre lo hemos dudado ${ }^{7}$, dado el pudor -al menos público, socialque siempre ha caracterizado a Barthes.

6 Queremos poner de relieve que la descripción que lleva a cabo de estas fotografías corresponde a la que ofrece de Daniel Boudinet al inicio de La Chambre claire.

7 Como dijimos en “L'espace autobiographique dans l'écriture de Roland Barthes” (2019: 371), del que desarrollamos las últimas páginas alusivas al diario dentro del espacio autobiográfico barthesiano. 
Dejamos de lado el bello texto "La lumière du Sud-Ouest", y "Au Palace ce soir...", que ya habían sido publicados. Ponemos de relieve, además, que observamos que no se encuentran en la carpeta de los archivos inéditos de Barthes relativos a Incidents.

Es cierto que los textos de "Soirées de Paris" - de agosto y septiembre de $1979^{8}-$ presentan un grado de composición que permitiría prever una publicación futura. Es cierto también que en uno de los textos de este conjunto, el correspondiente al 27 de agosto de 1979, Barthes anota sobre el manuscrito, al inicio de la página: "mettre au passé composé" (1987: 85), lo que podría confirmarlo.

No obstante, Barthes podría estar guardando - como creemos- este material reservado con vistas a una futura obra. De hecho, Barthes confiesa abandonar las "Soirées" "pour vérifier mes notes -et désormais tout écrire sur les fiches", tal como Wahl reconoce en su "Note de l'éditeur" (Barthes, 1987: 9).

Así, en los borradores que por el momento conocemos del proyecto de su obra Vita Nova (2002b: 1007-1018), contemporáneos por otra parte de los textos de "Soirées de Paris", Barthes hace alusión a la "drague", a las "vaines soirées", al gigolo -como a la panadera, después de todo-; encontramos igualmente la alusión al "enfant marocain" de los "Incidents", pero nunca podremos saber bajo qué forma definitiva estas experiencias se habrían convertido en Texto.

Las páginas de "Soirées de Paris" nos ofrecen también interesantes y trascendentes meditaciones literarias. En agosto de 1979 Barthes se encuentra leyendo las Mémoires d'outretombe de Chateaubriand, "le vrai livre", añadiendo "Toujours cette pensée: et si les modernes se trompaient? S'ils n'avaient pas de talent?” (1987: 80).

Los fragmentos de "Incidents", que abren la obra, pequeñas anotaciones, a veces simplemente detalles, como haïkus, de sus experiencias en Marruecos entre 1968 et 1969 -que Barthes hubiera podido publicar a lo largo de esos diez años, si lo hubiera deseado-, así como los textos de agosto-septiembre de 1979, que componen las "Soirées de Paris" hacen aparecer, y desvelan al mismo tiempo públicamente, los escritos íntimos relativos a las relaciones de Barthes con los jóvenes árabes, así como una homosexualidad vivida de forma lastimosa.

La publicación de Incidents par François Wahl -prematura para Antoine Compagnon, como expresa en su L'âge des lettres, por cuanto se trataba de los "textes inédits les plus privés de Roland" (2015: 123)-, y contestada igualmente por diversos autores, al modificar la imagen que se poseía de Barthes y desvelar lo que Barthes pretendía ocultar: su homosexualidad (Culler, 2015: 147 y 145), contará no obstante, como recuerda dicho autor, con la aceptación de Michel Salzedo (2015: 122), el hermano de Barthes. Sin embargo, cuando se plantea años más tarde la publicación de otros fragmentos de diarios llevados por Barthes, y

8 En la que aparece no obstante intercalado, con gran contraste de tono y temática, un fragmento de una noche en Urt con "Rachel et M." -Rachel, su cuñada, y su hermano Michel- "si affectueux, si naïf, si sensible à ce qui est beau, comme l'était mam." (1987: 89). El atardecer es delicioso: "Le crépuscule, déjà avancé, d'une beauté extraordinaire, presque étrange à force de perfection: un gris ouaté et léger pas triste, les bancs de brume au loin de l'autre côté de l'Adour, le chemin bordé de maisons paisibles pleines de fleurs, une demi-lune d'or, véritablement, des bruits de grillons, comme autrefois: noblesse, paix" (1987: 89). 
mucho menos comprometidos, Wahl, que no será esta vez el editor, contestará estas publicaciones y se desencadenará toda una polémica sobre el derecho de publicar los diarios íntimos de un autor?.

Estas publicaciones más recientes nos permiten conocer otros momentos de su diario de tono muy diferente entre ambos. Ya sea por su viaje a China, ya sea por el dolor ante la muerte de su madre, estos dos diarios presentan no obstante cada uno una gran unidad.

En 2009 se publica en coedición entre l'IMEC y la editorial Christian Bourgois, los Carnets $d u$ voyage en Chine. La edición es establecida y anotada par Anne Herschberg, y está compuesta de cuatro carnets - conteniendo el cuarto el índice y un índice temático-, que Barthes redactó entre el 11 de abril y el 4 de mayo de 1974, la duración exacta de su viaje a China.

Como los investigadores barthésianos saben, Barthes viajó a Chine en compañía de François Wahl y de ciertos amis del grupo Tel Quel, en la etapa maoísta: Philippe Sollers, Marcelin Pleynet, e incluso Julia Kristeva. Sobre este viaje Barthes escribirá y publicará en vida un artículo: “Alors, la Chine?" (1974), pero el conjunto del material -salvo algunas páginas manuscritas del primer Carnet, publicadas en $R / B$, la bella edición del catálogo de la exposición en el Centro Pompidou, entre noviembre de 2002 y marzo de 2003, bajo la dirección de Marianne Alphant y Nathalie Léger- (2002: 209-225), estos carnets permanecieron inéditos más de treinta años.

No obstante, Barthes aprovecha sus experiencias en la civilización china para hacer un paréntesis en el desarrollo previsto en el seminario que está impartiendo durante el curso1973-74 en la École Pratique des Hautes Études, e integra en la sesión del 8 de mayo, sólo cuatro días después de su vuelta a París, unas reflexiones, que califica de totalmente provisionales, sobre las condiciones posibles/o imposibles de un discurso sobre la China $\operatorname{popular}^{10}$.

Las primeras líneas de este diario desconciertan, y recuerdan desde nuestro punto de vista el diario de Paludes de Gide: "11 avril. Départ, lavé de pied en cap. Oublié de me laver les oreilles" (2009a: 19).

Además, algunas líneas después: “Orly. Retard. Ph. S. [por Philippe Sollers] achète un saucisson et du pain hors douane et nous croûtons dans le salon d'attente". Hablando de la cena en el avión: "riz grisâtre et graisseux -dont deux grains tombent immanquablement sur

9 Tal como la prensa de la época puso en evidencia, la oposición, esta vez, de François Wahl, a la publicación de estas anotaciones póstumas desencadenó toda una serie de acusaciones cruzadas, principalmente virulentas entre él y, por otra parte, Olivier Corpet (Director de l'IMEC), Bernard Comment, director ya de Seuil, y Éric Marty, editor de las Euvres complètes de Barthes y amigo de Michel Salzedo, hermano de Barthes y su derechohabiente.

10 Reproducidas en Roland Barthes, Le lexique de l'auteur, suivi de Fragments inédits du Roland Barthes par Roland Barthes (2010: 229-245). Si indicamos estas reflexiones como paréntesis de su seminario es porque el objeto del mismo es la preparación de los temas y el glosario de lo que será su próximo libro Roland Barthes par Roland Barthes. 
mon pantalon neuf' (2009a: 19), escribe Barthes no sin humor, o como prolepsis simbólica de un viaje, para él, algo malogrado.

El "Nous décollons. OUF" en mayúsculas y encuadrado, que Barthes expresa al regresar resulta muy significativo (2009: 213).

Barthes anota escrupulosamente en cada carnet el plan, de la mañana a la tarde, que habían previsto para ellos cada día. Hace de vez en cuando incluso algunos dibujos para recordar las situaciones. Anota las visitas a las fábricas, a la imprenta, la guardería, el hospital, la escuela de acupuntura, la Universidad de Pekín, la Escuela Normal Superior de Nankin, a su escuela primaria, a las comunas populares, les conversaciones. Habla abundantemente, como es natural, de la Revolución cultural proletaria, de la vida en China tras ella, de Mao (y de su caligrafía -siempre el significante-), de Lin Piao, Confucio, Marx, Hegel, Lenin, Trostsky, del revisionismo, del "verrouillage complet de l'information" (2009a: 183) Guarda datos, estadísticas, que apunta en sus carnets.

Nos da a conocer la organización del trabajo y de los estudios en la fábrica: "Mouvement d'études Marx Mao dans les larges masses des ouvriers de notre usine: 80 groupes d'études en dehors des heures de travail" (2009a: 30). "Pas de dimanche. Rotation" (2009a: 27).

Alude a las conversaciones con escritores, con los profesores de filosofía, con los lingüistas. Alude a la cultura, a los museos, los emplazamientos arqueológicos.

Para Anne Herschberg, estos carnets "Présentent une attention phénoménologique à la Chine de 1974, davantage intéressée par les gens et les choses, que par les musées et les sites archéologiques" (2009a: 9). Y es cierto. Resulta patente el desinterés de Barthes ante la tumba Ming: "Je reste dans l'auto, pendant que les autres sortent, photographient. Flemme. Envie de ville, de magasins, de café" (2009a: 95), o ante la de los Han Ouest (2000 años): "Étouffant. Attendu les autres dehors devant le tombeau, en même temps que cent cinquante personnes" (2009a: 113).

La muraille chinoise se despacha en dos líneas (2009a: 166, 173). Sólo la tumba del tercer emperador Ming le produce algún interés: "peut-être l'espace le plus beau que nous ayons vu: Pagodes, Pins, Palmiers, Courettes, portiques, etc. Bleu, rouge, vert" (2009a: 175). Frente a la historia, las sensaciones.

El diario ofrece así otra dimensión. Barthes se siente agotado. La multitud y los planes demasiado apretados le fatigan.

Sólo cuatro días después de su llegada, Barthes muestra ya su hastío frente a los discursos propagandísticos que deben soportar. Barthes se evade y su pensamiento se traslada a las hojas de su té:

Speech mortel, comparaison passé/présent. Recenser les topoï. Je regarde mon verre de thé: les feuilles vertes se sont largement épanouies et forment toute une épaisseur au fond du verre. Mais le thé est très léger, insipide, à peine une tisane, c'est de l'eau chaude (2009a: 42). 
Todo está codificado, nada sale de la norma. No hay matices, no hay lugar para el placer, para la sorpresa. Incluso el lenguaje se inserta en el estereotipo político: "Placage de stéréotypes révolutionnaires" (2009a: 138).

Barthes lo confiesa por otra parte durante su viaje en tren hacia Nankin: "Toutes ces notes attesteront sans doute, la faillite, en ce pays, de mon écriture (par comparaison avec le Japon). Je ne trouve, en fait, rien à noter, à énumérer, à classer" (2009a: 73). La migraña y la carencia sexual (2009a: 117) contribuyeron probablemente a ese sentimiento de desánimo y de fracaso a lo largo de la estancia.

Enumera sus carencias: "Ce dont je suis dépossédé: de café, de salade, de flirt" (2009a: 145), y continúa: “Le soir: très fatigué et découragé. Sentiment: j’en ai plein le dos (y compris des conversations entre nous)" (2009a: 145). Incluso la vitalidad verbal de su querido Sollers llega a fatigarle (2009a: 119, 173).

No sólo la migraña, el insomnio, o las agendas demasiado apretadas, sino también el estar siempre acompañado, guiado, incluso junto a innumerables turistas, agotan a Barthes.

Tal como habíamos observado ante la tumba Ming, también ante el Túmulo de uno de los emperadores Barthes se alejará para poder al fin estar solo. Dejando de lado bruscamente su descripción, Barthes escribe:

Les autres montent en haut du tertre. Je reste seul et m'assieds par terre dans un verger, au-dessus du champ de blé, devant l'horizon, vaste, léger, vert. Quelques bâtisses de briques d'un rose-beigé poudreux, une musique lointaine. Un champ brun beige, à gros sillons ondulés. Des arbres ici et là, au fond. Bruit d'un vélo moteur invisible.

Retour en auto à travers la belle campagne avec F.W. Moins de monde (2009a: 153).

Aun así, el diario que Barthes escribe en China nos permite observar que el humor y la ironía están bien presentes a lo largo de las anotaciones: "une douzaine de chinois en veste noire à col montant [...] On dirait un couvent qui se déplace” (2009a: 20). Tras jugar con el significante chino y el latinizado de Confucius, Barthes cambia repentinamente de registro: “Histoire du Bic usé dont j'essaye de me débarrasser, que je planque au fin fond d'un tiroir et qui me revient trois fois" (2009a: 135).

A la llegada a Pekín observa: "Jeunes soldats: l'impression de rien sous leur tunique. Sourires" (2009a: 21). Se pregunta en diversas ocasiones si la sexualidad existe en China (2009a: 22, 24).

Barthes se distrae con los juegos de palabras: "Lin Piao Confucius en France? Jusqu'à présent, confus en France, incompréhensible" (2009a: 63). Alude a las banderitas que hacen pender con motivo de sus visitas: "Bienvenue à Tel Quel" (2009a: 32). Barthes imagina lo inaudito de ello en Francia: "Chaque atelier: applaudissements. 'Tel quel et ses amis se font applaudir dans les usines de Chine"” (2009a: 32).

El semiólogo se desvela: “Coupes cheveux codées. Quelle impression! absence totale de mode. Degré zéro du vêtement” (2009a: 23). 
La atención al significante está presente: “Le signifiant: au fond: tout ce que j’aime et cela seulement" (2009a: 145); "Signifiant: ne pas y mettre le Vêtement; il est ici du côté du signifié" (2009a: 162). Observa la censura como un "refoulement du signifiant. Effacement du Texte au profit du Langage?” (2009a: 159); "[Le signifiant: plutôt que censure, dire: le "silenciment"] [La mise au silence]" (2009a: 160). "Censure du signifiant par le signe stalinien: affiches, tableaux [...], langage" (2009a: 173).

Uno de los intereses mayores de Barthes en el viaje es observar el estereotipo, buscar los intersticios de esas "briques" - como Barthes las denomina- de sentidos codificados que percibe por todas partes. Va a la búsqueda de los signos, pero no percibe más que el signo.

Pero hay un elemento que cobra en las líneas de los carnets un protagonismo esencial muy alejado del discurso político en el que se encuentran alojados a diario, y es la atención prestada a la naturaleza, como se ha dejado ver ya en fragmentos anteriores. De forma reiterada la naturaleza se ofrece ante Barthes como una evasión necesaria:

Histoire des cinq Dynasties. Grésil. Esclavagisme et Société féodale. Tableaux. Objets en bronze poussiéreux. Ça promet d'être fort ennuyeux. Mais dehors le jardin est charmant sous la pluie légère, très feullu avec des stèles, des éléments de Pagode à montants rouges, des pins, des rosiers -lieu plus français que japonais. Arbuste genre agave (il y en a à Urt) (2009a: 160).

Barthes se mostrará sensible ante la belleza de la naturaleza y la trasladará a los carnets, que cobran así una dimensión muy diferente de los objetivos sociopolíticos, etnográficos y culturales que poseían las anotaciones sobre este país. Los ejemplos podrían ser numerosos: “Cependant soleil, brise. Tout cela d'un charme... [...] Que restera-t-il de Pékin? Une brise, une lumière voilée, une tiédeur, ciel bleu léger, quelques flocons" (2009a: 23); "Jardin. Pruniers japonais, magnolias en fleurs. Rocaille" (2009a: 23); "Hôtel: dans un jardin-parc, avec de belles essences d'arbres, magnolias, platanes, sapins. Très français” (2009a:74); Los "Parcs à Pivoines" (2009a: 112-3); "Plus chaud. Palmiers. Mimosas. Parfum" (2009a: 35); “Jardins. Campagne. Sur le côté du Jardin, des iris. Il fait beau” (2009a: 137).

Los colores - tan importantes en sus textos sobre el Sud-Ouest- aparecen con insistencia: "Drapeaux rouges. Brecht. Chercher la Couleur -resaltamos la cursiva del término-. Gris bleus. Taches rouges. Fer. Kaki. Vert” (2009a: 22); “Quelles couleurs! passées, un peu de jaune, de rouille passé, du beige, du touron...” (2009a: 36-37).

Pero también los olores -"Fruits (pommes, raisins, poires. Forte odeur)" (2009a: 26)-, y los sabores, del té y del café aguados, pero también de las comidas, de los alimentos - “Toujours un excellent dîner: brochettes très sèches (de mouton), canard laqué (avec crêpes, tiges d'oignons jeunes), beignets caramélisés de pomme" (2009a: 183); "Délicieux beignets de langoustines, moelleux, goût de farine. Mélange de sel et d'aromates (Thym, peut-être Cannelle). Anguilles du Yang Tse à l'ail. Raviolis à la vapeur, dans un grand tambourin en bois 
[...] Pain à la vapeur, frit, croustillant: délicieux. Et un bon cigare" (2009a: 176)- forman parte de estos carnets sobre su viaje a China.

La naturaleza no está ausente, y otorga un toque de frescor, de vida. Incluso tras haber traído a colación el discurso de una mujer criticando a Confucio y Lin Piao, Barthes escribe en la línea siguiente: “Quelques pots de géranium” (2009a: 27).

Olores, colores, sabores. En China, también, el recuerdo pasa por el cuerpo.

El segundo texto, publicado también en 2009, del diario íntimo e inédito de Barthes, es Journal de deuil. Barthes lo inicia el 26 de octubre de 1977, el día posterior a la muerte de su madre, y se prolongará hasta el 15 de septiembre de 1979.

Las 330 fichas que componen este Journal de deuil forman parte de esas casi 13.000 fichas que, como pone en evidencia Nathalie Léger -conservadora en el IMEC y responsable de la edición del diario en 2009-, Barthes escribió desde 1943 en el Sanatorio de Saint-Hilaire du Touvet hasta su muerte, pero, mientras que él solía reclasificar a menudo las fichas, esta parte del fichero se abre y se cierra sobre ella misma. La mayor parte están fechadas y se siguen formando una unidad (2009: 84-85).

Su hermano, Michel Salzedo, concedió la autorización para la publicación de estas notas, que dan muestra "plus de l'intériorité que de l'intimité, de la méditation que de la confidence" (2009: 86), lo que no impide que nos encontremos ante un verdadero diario íntimo.

Valérie Stiénon y Laurent Demoulin tienen razón en observar que ambas recopilaciones de fragmentos, aun escritos en momentos alejados y circunstancias diferentes, no son en absoluto discordantes: "L'écriture du deuil et l'écriture du voyage manifestent en commun les ambiguiités de l'image de soi, la prédilection pour le fragmentaire, l'expression de la solitude et la lutte de la nuance contre le conformisme" (2009: 3).

Barthes escribe casi todos los días. Ya sea en París o en Urt. Incluso varias fichas el mismo día. Las anotaciones en el diario son breves. En ocasiones una única línea ocupa la ficha. Barthes traslada el sentimiento del instante. Escritura fragmentaria, concisión; las pequeñas frases son como haïkus. Barthes no consigue superar la pena, "le chagrin", término proustiano que él prefiere frente al de "deuil" (2009b: 83) ${ }^{11}$, pero al mismo tiempo parece complacerse en ese dolor, como si de esa forma estuviera más cerca de su madre, como si pudiera así vivir todavía con ella. Es el lamento por la ausencia de la madre, pero también por la compañera, con la que habrá vivido más de sesenta años.

El 31 de julio de 1978, escribe -esta vez en lápiz'²: “J’habite mon chagrin et cela

11 A pesar de que irá alternando los dos términos a lo largo de los fragmentos, y que el título que decidirá es Journal de deuil.

12 Agradecemos a Marie-Odile Germain, responsable del "Fonds Barthes" en la BnF, y a Éric Marty, y a través de él a Michel Salzedo, la oportunidad brindada para consultar las fichas manuscritas relativas a esta obra. Las fichas, en medias cuartillas de DIN A-4, son escritas a mano, normalmente en tinta azul no oscura y líneas bastante espaciadas. 
me rend heureux". Y un poco más abajo, también en lápiz: “Tout m’est insupportable qui m'empêche d'habiter mon chagrin" (2009b: 185). Con la misma fecha, pero en ficha diferente, leemos también: "je ne souhaite rien d'autre que d'habiter mon chagrin" (2009b: 186). No soporta una distracción, lo que asumiría como "une substitution de mam" (2009b: 206)13.

Barthes nos transmite perfectamente la sensación "du définitif", esa sensación de descubrir que "mam. n'est plus là à jamais" (2009b: 192)14. Sus frases nos penetran: "savoir que mam. est morte à jamais, complètement" (2009b: 130); "J'affronte la longue série des temps sans elle" (2009b: 48).

Journal de deuil es, como su título indica, un diario sobre su duelo, a pesar de que Barthes se distancia sustituyendo el determinante contracto -que supondría la inscripción en alguien- por la preposición. El diario no focaliza sobre la madre, de la que no nos ofrece momentos vividos con ella, sino sobre su propio dolor ante su ausencia definitiva.

Maïté Snauwert resalta, acertadamente, "la dimension physique de son expérience" (2013), la corporalidad en el dolor. Lo que no es extraño, pues la pulsión del cuerpo ha acompañado habitualmente la escritura de Barthes. La emotividad, el llanto estarán presentes en muchos fragmentos. La muerte de su madre le dará además la certitud, hasta ese momento abstracta, de la mortalidad del hombre, de su propia muerte (2009b: 219).

Para Antoine Compagnon se trata de "l'autoanalyse d'un deuil, aussi intense que dans Albertine disparue" "Barthes, comme le héros de Proust, vit l'angoisse -la culpabilité- de l'atténuation de l'émotion"' (2013).

Barthes citará de hecho a Proust en su Journal de deuil, concretamente el Contre Sainte-Beuve, del que escribirá ciertos fragmentos en su diario el 10 de agosto de 1978 queremos poner de relieve que en dicha fecha Barthes escribe cinco fichas. El dolor se hace literario. Barthes hará alusión a la dulzura del rostro de la madre de Proust, a su bondad, a las páginas desgarradoras sobre la separación de Proust y de su madre, al retrato por Proust de la abuela de Robert de Flers, que acaba de morir, y, además, a los versículos de Jesús resucitando a Lázaro (2009b: 197-199). Al final de ese mismo mes, Barthes recordará además el retrato conmovedor que Gide hizo de Madeleine (2009b: 208).

En realidad Barthes y su madre hablaban poco, salvo el "Je suis là" (2009b: 47, 50, 123), que se lanzaban a menudo. Barthes reconoce que él permanecía a menudo en silencio. La palabra compartida era la simple presencia (2009b: 200 y 202). Su hermano, asombrosamente sincero en la entrevista del Coloquio "Avec Roland Barthes", en el Collège de France, confiesa también haber hablado muy poco con Roland (Salzedo. 2016), pero ese silencio es como un satori zen.

Barthes aludirá en diversas ocasiones en su diario a la acedia ${ }^{15}$-incluida la amorosa-

13 Barthes había ya utilizado esta abreviatura al aludir, en los Carnets du voyage en Chine, al telegrama recibido y a las "deux lettres de mam" (2009a: 177 y 169).

14 Ver también p. 21, 88 y 124.

15 La "acédie" será al mismo tiempo uno de los temas tratados por Barthes en su curso del Collège de France sobre 
que lo envuelve: "J'éprouve -et c'est dur- la sécheresse de coeur-, l'acédie" (2009b: 129), a esa amargura del corazón que sigue sin disminuir a finales de 1978 (2009b: 230). "Désarroi, déshérence, apathie" (2009b: 69), "désaffection de la mondanité" (2009b: 86), "blocage" (2009b: 90), "malaise", “détresse" (2009b: 97), "tristesse nauseuse" (2009b: 106) son algunos de los términos con los que Barthes expresa su estado de ánimo.

La acedia, consecuencia de la desolación (2009b: 190-191), se encuentra bien presente en las fichas, pero poco a poco, sobre todo a partir de marzo de 1978, el proyecto de un texto sobre su madre se perfila. El 23 de marzo de ese año Barthes alude ya al futuro libro:

“Hâte que j'ai (sans cesse vérifiée depuis des semaines) de retrouver la liberté (débarrassé des retards) de me mettre au livre sur la Photo, c'est-à dire d'intégrer mon chagrin à une écriture" (2009b: 114).

El 12 abril expresa ya el deseo de realizar, contra el olvido, el "monumento" (2009b: 125), y el 5 de junio insiste en el monumento, en "faire reconnaître mam." (2009b: 145).

Barthes comenzará el 11 de junio de 1978 (2009b: 151) a mirar las fotos de su madre. Dos días antes, el 9 de junio, Barthes atraviesa la iglesia Saint-Sulpice, cercana a su casa, se sienta un segundo, y como nos confiesa, realiza una "sorte de 'prière' instinctive: que je réussisse le livre Photo-Mam." (2009b: 148). El 13 confiesa sentirse "bouleversé par une où mam. petite fille, douce, discrète, à côté de Philippe Binger (Jardin d'hiver de Chennevières, 1898)" (2009b: 155) $)^{16}$.

Es "l'accession du Chagrin à l'Actif", como testimonia el 6 de octubre: "Liquider d'arrache-pied ce qui m'empêche, me sépare d'écrire le texte sur mam.: le départ actif du Chagrin: l'accession du Chagrin à l'Actif' (2009b: 217).

La muerte de la madre, como en Proust, desencadena la mutación en la vida y la escritura. El 17 de setiembre confiesa: "Depuis la mort de mam. [...] effort acharné pour mettre en oeuvre un grand projet" (2009b: 213), proyecto también centrado en el "vouloir-écrire", en su acercamiento liberado hacia lo "romanesque".

En diciembre será todavía más claro: "Sans doute je serai mal, tant que je n'aurai pas écrit quelque chose à partir d'elle (Photo, ou autre chose)" (2009b: 227). Los lectores reconocerán evidentemente que se trata de La chambre claire $^{17}$.

Las anotaciones del diario se espacian a lo largo de 1979. Si Barthes ralentiza e inclu-

"La préparation du roman”, ya en la sesión inaugural del 2 de diciembre de 1978 (2015: 20), y, casi en estructura circular, en la sesión del 9 de febrero de 1980, en la que vuelve sobre ese "ennui", "perte de pulsión", "paresse noire" (2015: 498). El "deuil", la "acédie" forman parte precisamente del proyecto de Vita Nova (2002b: 10081018).

16 Hecho que Barthes sitúa en noviembre en La Chambre claire (2002: 841).

17 Los diferentes títulos que Barthes baraja para la obra y que, tras la consulta de los manuscritos, Nachtergael nos da a conocer, son a nuestro entender muy significativos, y se alejan aún más de la alusión técnica a la fotografía por un acercamiento mucho más personal "Ma photographie" "La photographie: plaisir et deuil" "À la Recherche de la photographie" (2012: 120). Curiosamente, como Nachtergael nos recuerda, la fotografía de la abuela fallecida del narrador de la Recherche y la de la madre de Barthes niña en el "Jardin d'Hiver" son realizadas en la misma época 1897-98 (2012: 125). 
so deja el diario en setiembre no es porque el dolor haya desaparecido, o incluso se debilite, es porque la madre se hace texto.

El 29 de marzo de 1979 Barthes hará alusión por última vez al "monumento" en un fragmento fundamental: "je vis sans aucun souci de la postérité [...] aucune envie de "monument" - mais je ne peux supporter qu'il en soit ainsi pour mam. (peut-être parce qu'elle n'a pas écrit et que son souvenir dépend entièrement de moi" (2009b: 245).

Barthes ha encontrado el monumento, va a lograr el monumento, aunque se trate todavía de un pequeño monumento, y es, como ya se ha puesto en evidencia, La chambre claire $^{18}$, en concreto la segunda parte de La chambre claire, a través de la foto de su madre, niña, la foto del Jardin d'hiver.

Barthes no nos permitirá conocer la foto. La deja sólo para él, pero en su juego oblicuo nos ofrece su avatar. Es la foto "La Souche", perteneciente, como se indica, a la colección del autor, y que aparece en la obra muchas páginas después (1980: 163). La madre, niña, tendrá la misma pose y dulzura.

El capítulo posee tintes netamente proustianos desde su inicio:

Or un soir de novembre, peu de temps après la mort de ma mère, je rangeai des photographies. Je n'espérais pas la "retrouver", je n'attendais rien de "ces photographies d'un être devant lesquelles on se le rappelle moins bien qu'en se contentant de penser à lui" (Proust). Je savais bien que, par cette fatalité qui est l'un des traits les plus atroces du deuil, j'aurais beau consulter des images, je ne pourrais jamais plus me rappeler ses traits (les appeler tout entiers à moi) [...]" (1980: 99). V 841).

La foto consigue "retrouver" (1980: 101) sa mère, su "résurrection" (1980: 101), llegar a su esencia:

J'observais la petite fille et je retrouvai enfin ma mère. La clarté de son visage, la pose naïve de ses mains, la place qu'elle avait occupée docilement sans se montrer ni se cacher, son expression enfin [...] la figure d'une innocence souveraine [...] l'affirmation d'une douceur. Sur cette image de petite fille je voyais la bonté qui avait formé son être tout de suite et pour toujours (1980: 107).

Incluso la primera parte, la del "“savoir' photographique” (1980: 17), comienza, a guisa de preparación, como un cuento: "Un jour, il y a bien longtemps, je tombai sur une photographie du dernier frère de Napoléon [...]" (1980: 13) ${ }^{19}$. Es el inicio del libro y el intertexto proustiano no se le escapa a nadie. Proust está cada vez más presente ${ }^{20}$, y Barthes introduce desde el incipit el "romanesque" cuya textualidad está buscando.

18 Cuya redacción, como Barthes indica al final del libro, se lleva a cabo entre el 15 de abril y el 3 de junio de 1979.

19 Incluso el manuscrito, que también hemos tenido la oportunidad de consultar, aunque difiere de la escritura definitiva, mantiene la referencia literaria: "Depuis longtemps, je m'intéressais à la Photographie. J'avais décrété [...]" (La Chambre claire Ms 1. NAF 28630, I, 11 Roland Barthes).

20 Recordemos la importante conferencia de 1978 "Longtemps je me suis couché de bonne heure", y que el último 
Como hemos podido comprobar, el diario que Barthes escribe tras la muerte de su madre se convierte así en el motor impulsor para escribir La Chambre claire, la puerta que ha permitido, tras la publicación de los diarios, acceder a la gestación de la obra.

Este libro es el texto magnífico sobre la fotografía, sobre el punctum, pero es sobre todo para Barthes el libro Photo-Mam.

Como hemos podido comprobar, el diario de Gide fue el motor impulsor de la escritura diarística barthesiana, diario que comenzó durante su estancia en el Sanatorio, con un carácter bibliográfico, y al que se fueron sumando diferentes anotaciones de carácter diverso en fichas a lo largo de su vida.

El diario de Gide siguió también acompañando el desarrollo de La préparation du roman, botón de muestra del proyecto reflexivo de plasmación del "romanesque" al que se acercaba Barthes a tientas, pero progresivamente, en los últimos años, y será precisamente este diario fragmentario, diario de sus relaciones masculinas, en Marruecos o en París; diario de la enfermedad de la madre y de Urt; diario de la muerte de la madre, que no sólo se convertiría en el monumento de La Chambre claire, sino que todo ello iba a materializarse de forma textual en uno de los proyectos últimos de Barthes: su futura Vita Nova.

\section{Referencias bibliográficas}

Alphant, Marianne \& Nathalie LÉGer. 2002. R/B. (Catalogue de l'exposition au Centre Pompidou, novembre 2002-mars 2003). Paris, Seuil/Centre Pompidou/IMEC.

BARThes, Roland. 1942. "Notes sur André Gide et son journal” in Existences, n 27, juillet 1942.

BARThes, Roland. 1974. “Alors, la Chine?”, Le Monde, 24 mai 1974.

BARTHes, Roland. 1975. Roland Barthes par Roland Barthes. Paris, Seuil (coll. Écrivains de toujours).

Barthes, Roland. 1980. La Chambre claire. Paris, Cahiers du cinéma/Gallimard/Seuil.

Barthes, Roland. 1987. Incidents (éd. François Wahl). Paris, Seuil.

BARThes, Roland. 2002a, "Délibération". Tel Quel, hiver 1979. Repris dans Euvres complètes V: 1977-1980 (éd. Éric Marty), Paris, Seuil, 668-681.

BARTHES, Roland. 2002b. "Transcription de Vita Nova". Euvres complètes V: 1977-1980 (éd. Éric Marty). Paris, Seuil, 1007-1018.

Barthes, Roland. 2009a. Carnets du voyage en Chine (éd. Anne Herschberg Pierrot). Paris, Christian Bourgois/Imec.

Barthes, Roland. 2009b. Journal de deuil (éd. Nathalie Léger). Paris, Seuil/Imec.

curso que Barthes había proyectado impartir en el Collège de France para la primavera de 1980 se hubiera centrado en las fotografías del mundo de Proust. 
Barthes, Roland. 2010. Le lexique de l'auteur, suivi de Fragments inédits du Roland Barthes par Roland Barthes (éd. Anne Herschberg Pierrot), Paris, Seuil.

BARThes, Roland. 2015. La préparation du roman. Cours au Collège de France 1978-79 et 1979-80 (Texte annoté par Nathalie Léger. Transcription des enregistrements par Nathalie Lacroix et Avant-propos de Bernard Comment). Paris, Seuil.

Benito Fernández, José. 2011. Gide/Barthes. Cuaderno de niebla. Barcelona, Montesinos (Ediciones de Intervención cultural).

Compagnon, Antoine. 2013. "Écrire le deuil" in Acta fabula, vol. 14, n 2: "Let's Proust again!", février 2013. <http://www.fabula.org/revue/document7574.php.>

Compagnon, Antoine. 2015. L'âge des lettres. Paris, Gallimard-nrf.

Culler, Jonathan. 2015. Roland Barthes. Paris, Presses universitaires de Vincennes.

Genette, Gérard. "Le journal, l'antijournal” in Poétique, nº 47, 1981.

LÉGER, Nathalie. 2009. “Chaque fiche est une figure du chagrin”. Entretien avec Valérie

MARIN LA MesLÉE in Magazine littéraire, nº 482, janvier 2009, 84-85.

MARTY, Éric. 2007. "Roland Barthes et la question du genre. Le journal” in BARONI, Raphaël \& Marielle MACÉ (dir.). Le savoir des genres, La licorne, nº 79, P.U. de Rennes, 85-95.

Nachtergael, Magali. 2003. "Préface" à Roland Barthes, La préparation du roman I et II. Cours et séminaires au Collège de France (1978-1979 et 1979-1980). Paris, Seuil /IMEC.

Nachtergael, Magali. 2012. "Vers l'autobiographie New Look de Roland Barthes. Photographies, scénographie et réflexivité théorique" in Image \& Narrative, Vol. 13, No 4, 112-128.

Nachtergael, Magali (éd.). 2015. Roland Barthes, La préparation du roman I et II. Cours et séminaires au Collège de France (1978-79 et 1979-80). Paris, Seuil.

Salzedo, Michel. 2016. "Barthes, l'homme secret" (interview). Colloque

Nachtergael, Magali. 2012. “Avec Roland Barthes”, Collège de France, 13 et 16 janvier 2016. <https://www.college-de-france.fr/site/antoine-compagnon/Barthes-un-heritage-problem 6.htm>

Samoyault, Tiphaine. 2015a. Roland Barthes. Paris, Seuil (coll. Fiction \& Cie).

Samoyault, Tiphaine. 2015b. "Roland Barthes, une vie. Entretien avec Tiphaine Samoyault" (par Olivier BARBARANT et Nicolas Dutent) in Lettres françaises, $\mathrm{n}^{\circ} 127,11$ juin 2015, 2-4. $<$ https://www.humanite.fr/sites/default/files/medias/2015/06/20150611_011f_127web_0. pdf $>$

SiRvent Ramos, Ángeles. 2019. “L'espace autobiographique dans l'écriture de Roland Barthes", in Figuerola, Carme (ed.), Evocar la literatura francesa y francófona de la modernidad. Homenaje a Angels Santa, Ediciones de la Universitat de Lleida / Pagès Editors, 361-376.

SNAUWAERT, Maïté. 2013. "Physique du deuil. Note sur la dernière vie de Roland Barthes" 
Anales de Filología Francesa, n. ${ }^{\circ}$ 27, 2019

LOS DIARIOS DE ROLAND BARTHES

in Temps zéro, "incursion” du 31 mars 2013 [en ligne]. <http://tempszéro.contemporain.info/ document $865>$

StiÉnon, Valérie \& Laurent Demoulin. 2009. "Roland Barthes, ethnographe de lui-même. Fiches, carnets et notes inédites" in Culture, Université de Liège.

$<$ http://culture.ulg.ac.be/jcms/prod_63596/roland-barthes-ethnographe-de-lui-meme> 